BOSQUE 16(1): 69-75, 1995

\title{
Nutrientes minerales y crecimiento de árboles abonados de Eucalyptus grandis en el Estado de São Paulo
}

\author{
Mineral nutrients and growth of fertilized Eucalyptus grandis in the State of São Paulo, Brazil
}

C.D.O.: 181.32

ANTONIO F.J. BELLOTE, CARLOS A. FERREIRA

Embrapa/CNP Florestas, Caixa Postal 319 - ColombolPR - CEP 83411-000-Brasil.

\section{SUMMARY}

This paper discusses the relationship between nutrients concentration in the leaves and soil, as well as tree growth in Eucalyptus grandis plantations. The survey was carried out in five locations in the Cerrado region of the State of São Paulo, Brazil. It was concluded that the trees were adequately nourished with $\mathrm{S}$, Fe, Mn and $\mathrm{Zn}$; $\mathrm{P}$ concentrations in the leaves were below the optimum reported in literature; $\mathrm{K}$ and $\mathrm{Mg}$ were the nutrients that most seriously limited tree growth, in ali locations despite fertilization; concentrations of 2.6 and $7.5 \mathrm{mg} / \mathrm{g} \mathrm{D} . \mathrm{M}$. of $\mathrm{Mg}$ and $\mathrm{K}$ were adequate leveIs for both nutrients; fertilization with $\mathrm{N}, \mathrm{P}, \mathrm{K}$ and $\mathrm{Mg}$ can increase the growth of the trees in allocations; $\mathrm{Ca}$ and $\mathrm{B}$ concentrations in the leaves were not related to the growth of the trees, showing a dilution effect.

\section{RESUMEN}

Este trabajo fue desarrollado con el objetivo de identificar los elementos minerales limitantes aI crecimiento de Eucalyptus grandis plantado en distintos sitios. Para su ejecución fueron colectadas muestras de tejido vegetal y suelo, en plantaciones con 3 afios de edad, en 5 sitios deI Estado de São Paulo, Brasil. Los resultados obtenidos permitieron las siguientes conclusiones: las plantaciones de Eucalyptus en las regiones estudiadas se encuentran adecuadamente suplidas de S, Fe, Mn y Zn; los contenidos de $\mathrm{P}$ en las hojas se encuentran bajo el valor óptimo reportado en la bibliografía; $\mathrm{K}$ y Mg son los nutrientes minerales que limitan más significativamente el crecimiento de los árboles en todos los sitios, también en plantaciones fertilizadas; árboles cuyas hojas presentan contenidos de 2.6 y $7.5 \mathrm{mg} / \mathrm{g}$ en la materia seca de $\mathrm{Mg} \mathrm{y} \mathrm{K}$, respectivamente, están adecuadamente nutridos de estos elementos; el aumento en la oferta de $\mathrm{N}, \mathrm{P}, \mathrm{K}$ Y Mg en el suelo, a través de una fertilización, puede aumentar el crecimiento de los árboles en todos los sitios; los contenidos de $\mathrm{Ca}$ y $\mathrm{B}$ en las hojas no están relacionados con el crecimiento de los árboles, mostrando incluso efecto de dilución.

\section{INTRODUCCION}

Eucalyptus es el género más plantado en el Estado de São Paulo. Aunque la productividad media en plantaciones con elevado nivel de tecnología en el Estado es de 40 nr'/ha-aíto, ésta es muy variable, existiendo áreas con productividad desde 19 hasta $72 \mathrm{~m}^{3} /$ ha-afio (Bellote, 1990).

Entre los factores responsables de esta variación está el uso generalizado de la misma formulación de fertilizantes minerales en extensas áreas, sin considerar las variaciones de los suelos. Esto muestra la inexistencia de criterios adecuados para el uso de fertilizantes, pudiendo en determinados sitios incluso resultar en una utilización inadecuada de ciertos elementos minerales.

Las recomendaciones de fertilizantes minerales se basan en nitrógeno, fósforo y potasio, pero muy raramente a calcio, boro y zinco Actualmente, la mayoría de las plantaciones comerciales son fertilizadas con utilización de prácticamente la misma formulación N-P-K, independiente de la especie, tipo de suelo y época de plantación. Las dosis recomendadas en trabajos de Garlipp y Balloni (1980b), Novais eta!. (1980), Barros eta!. (1981), Gonçalves y Diniz (1981) son de 20 hasta $64 \mathrm{~kg}$ 
$\mathrm{N} /$ ha de nitrógeno, 40 hasta $200 \mathrm{~kg} \mathrm{Pp}_{5} / \mathrm{ha}$ para el fósforo, y de 24 hasta $80 \mathrm{~kg} \mathrm{Kp} / \mathrm{ha}$ para el potasio. Como fuente de esos nutrientes han sido normalmente utilizados: sulfato de amonio (21\% $\mathrm{N})$, superfosfato simple $\left(21 \% \mathrm{P}_{2} \mathrm{O}_{5}\right)$, superfosfato triple $\left(45 \% \mathrm{P}_{2} \mathrm{0}_{5}\right)$ Y cloruro de potasio $(60 \% \mathrm{KP})$.

$\mathrm{La}$ fertilización con micronutrientes, específicamente $\mathrm{B}, \mathrm{Cu}$ y $\mathrm{Zn}$, es sin embargo bastante indefinida. Algunos trabajos demuestran que la aplicación de estos elementos es necesaria (Carvalho et al., 1978; Garlipp y Balloni, 1980a), mientras que otros indican no tener respuestas deI eucalipto para estos nutrientes (Defelipo et al. 1979; Barros et al. 1981).

Aparentemente, el empirismo que ha caracterizado la experimentación en nutrición de especies forestales en Brasil, a veces sin la necesaria caracterización de los suelos, hace que sea urgente el establecimiento de criterios de evaluación de la fertilidad deI suelo compatible con la especie, niveles de nutrientes minerales en el suelo y en los árboles.

Este trabajo fue desarrollado con el objetivo de identificar los elementos minerales limitantes aI crecimiento de Eucalyptus grandis plantado en distintos sitios. Para su ejecución fueron colectadas muestras de tejido vegetal y de suelo, en plantaciones con 3 afies de edad, ubicadas en 5 regi ones deI Estado de São Paulo. Son evaluadas las relaciones deI contenido de nutrientes minerales en las hojas de los árboles y en el suelo, con el crecimiento de los árboles en plantaciones abonadas.

\section{MATERIAL Y METODOS}

Para la obtención de las muestras utilizadas en este trabajo fueron seleccionados 15 sitios representativos de plantaciones comerciales de $E$. grandis Hill ex-Maiden, con 3 afios de edad, plantados en 5 regiones en el Estado de São Paulo, con espaciamiento de $3 \times 2 \mathrm{~m}$. Los sitios fueron abonados con N-P-K aI establecer la plantación, de acuerdo con el cuadro I. En cada sitio fueron seleccionados 15 árboles deI estrato dominante, de los cuales fueron recolectadas hojas deI tercio media de la copa, en el verano, de acuerdo con metodología propuesta por Bellote (1990). Cada conjunto de 3 árboles constituyeron una muestra, formando un total de 5 muestras compuestas por sitio.

Además de las hojas, fueron recolectadas muestras de suelo en las profundidades 0-10, 10-20 y 20-30 em, También fue evaluada la distribución deI sistema radicular en las tres profundidades. En base a las observaciones de la distribución radicular, fueron propuestos criterios para evaluar la media ponderada que mejor representara la oferta de nutrientes para los árboles en los primeros $30 \mathrm{em}$ deI suelo. Los datos obtenidos fueron correlacionados con el crecimiento de los árboles y la mejor ponderación fue obtenida en la relación 5:3:2. Las medias ponderadas de los contenidos de elementos minerales en el suelo fueron calculadas, para fines de análisis en este trabajo, por la siguiente fórmula:

Contenido deI elemento en el suelo $=0 . s^{*} A+0.3 * 8+0,2 * \mathrm{C}$

\section{CUADRO I}

Tipos de sueIo y fertilización aI plantar en los sitios de E. grandis utilizados, en el Estado de São Paulo, Brasil. Soil typesand fertilizatiomf $E$. grandis plantations in differentsites.

\begin{tabular}{llccc} 
& & \multicolumn{3}{c}{ Fertilización (kg/ha) } \\
Municipio & Suelos & N & Pps & Kp \\
Mogi-Guaçu & Latosol rojo amarillo* & 55 & 110 & 55 \\
Casa-Branca & Latosol rojo amarillo & 55 & 110 & 55 \\
ltirapina & Arenas Cuarzosas** & 29 & 90 & 29 \\
Itatinga & Arenas Cuarzosas & 33 & 95 & 20 \\
Angatuba & Arenas Cuarzosas & 20 & 148 & 20
\end{tabular}

* OrthicFerralsolde acuerdocon la FAO,

** FerralicArenosolde acuerdocon la FAO, 
donde:

$\mathrm{A}=$ Contenido deI elemento en el suelo (profundidad de $0-10 \mathrm{~cm}$ )

$\mathrm{B}=$ Contenido deI elemento en el suelo (profundidad de $10-20 \mathrm{~cm}$ )

$\mathrm{C}=$ Contenido deI elemento en el suelo (profundidad de 20-30 em)

Para los análisis químicos se utilizó extracción total de los macro y micronutrientes $(\mathrm{N}, \mathrm{P}, \mathrm{K}, \mathrm{Ca}$, $\mathrm{Mg}, \mathrm{S}, \mathrm{B}, \mathrm{Fe}, \mathrm{Mn}, \mathrm{Zn})$ y AI de la materia seca (M.S.) de las hojas. Los cationes cambiables K, $\mathrm{Ca}, \mathrm{Mg}, \mathrm{H}$, AI deI suelo fueron extraídos con solución $0.5 \mathrm{~N}$ de $\mathrm{NH}_{4} \mathrm{CI}$ (Trüby y Aldinger, 1989), el P con solución IN de ácido Cítrico (MolI, 1964) y la materia orgánica con solución IN de dicromato de potasio.

Los datos fueron analizados por regresión simpIe y regresión múltiple. Los modelos matemáticos resultantes de los análisis estadísticos fueron sometidos a pruebas de linearidad, multicolinearidad, heterocedasticidad y autocorrelación.

\section{RESULTADOS Y DISCUSION}

En el cuadro 2 son presentados la altura media de los árboles y los contenidos medios de nutrientes minerales en las hojas de los árboles, en las diferentes regiones deI estudio. En el cuadro 3 se encuentran los coeficientes de correlación entre la altura de los árboles, los elementos minerales en las hojas y en el suelo. Los análisis de regresión múltiple presentan bajo grado de tolerancia (multicolinearidad). Esta significa que existe correlación entre las variables independientes deI modelo matemático.

Los resultados, en el cuadro 3, muestran que los contenidos de $\mathrm{Ca}, \mathrm{S}, \mathrm{Fe}, \mathrm{Mn}$ y $\mathrm{Zn}$ en las hojas

CUADRO 2

Altura de los árboles, contenidos medi os de macronutrientes (mg/g M.S.) y micronutrientes (ug/g) en las hojas de los árboles, en las distintas regiones deI estudio.

Average height, macro (mg/g D.M.) and micronutrient (ug/g D.M.) content of leaves, for trees of E. grandis growing in the different sites.

\begin{tabular}{|c|c|c|c|c|c|}
\hline Variables & Mogi-Guaçu & Angatuba & Casa-Branca & Itatinga & Itirapina \\
\hline Altura (m) & $\begin{array}{l}20.3 \\
(2.1)\end{array}$ & $\begin{array}{l}17.7 \\
(1.6)\end{array}$ & $\begin{array}{l}16.8 \\
(0.8)\end{array}$ & $\begin{array}{l}15.9 \\
(1.2)\end{array}$ & $\begin{array}{l}1504 \\
(I A)\end{array}$ \\
\hline Nitrógeno & $\begin{array}{c}23.2 \\
(204)\end{array}$ & $\begin{array}{l}21.7 \\
(3.2)\end{array}$ & $\begin{array}{l}1904 \\
(1.9)\end{array}$ & $\begin{array}{l}19.9 \\
(3.8)\end{array}$ & $\begin{array}{l}18.0 \\
(1.6)\end{array}$ \\
\hline Fósforo & $\begin{array}{c}1.02 \\
(0.11)\end{array}$ & $\begin{array}{c}1.08 \\
(0.09)\end{array}$ & $\begin{array}{c}0.81 \\
(0.06)\end{array}$ & $\begin{array}{c}0.91 \\
(0.10)\end{array}$ & $\begin{array}{c}0.79 \\
(0.10)\end{array}$ \\
\hline Potasio & $\begin{array}{c}6.3 \\
(1.1)\end{array}$ & $\begin{array}{c}4.4 \\
(1.3)\end{array}$ & $\begin{array}{c}4.1 \\
(004)\end{array}$ & $\begin{array}{c}3.8 \\
(0.5)\end{array}$ & $\begin{array}{c}3.3 \\
(0.5)\end{array}$ \\
\hline Caleio & $\begin{array}{c}3.1 \\
(1.0)\end{array}$ & $\begin{array}{c}5.2 \\
(0.7)\end{array}$ & $\begin{array}{c}3.2 \\
(004)\end{array}$ & $\begin{array}{c}3.9 \\
(0.5)\end{array}$ & $\begin{array}{c}5.0 \\
(0.6)\end{array}$ \\
\hline Magnesio & $\begin{array}{c}2.5 \\
(004)\end{array}$ & $\begin{array}{c}1.9 \\
(0.2)\end{array}$ & $\begin{array}{c}1.7 \\
(0.3)\end{array}$ & $\begin{array}{c}1.7 \\
(0.2)\end{array}$ & $\begin{array}{c}1.5 \\
(0.2)\end{array}$ \\
\hline Zinc & $\begin{array}{l}12.3 \\
(2.2)\end{array}$ & $\begin{array}{c}8.8 \\
(2.3)\end{array}$ & $\begin{array}{c}8.0 \\
(2.3)\end{array}$ & $\begin{array}{c}9.8 \\
(1.7)\end{array}$ & $\begin{array}{l}10.0 \\
(1.9)\end{array}$ \\
\hline Boro & $\begin{array}{l}17.0 \\
(3.0)\end{array}$ & $\begin{array}{c}20.8 \\
(3.3)\end{array}$ & $\begin{array}{c}23.3 \\
(604)\end{array}$ & $\begin{array}{c}26.2 \\
(3.8)\end{array}$ & $\begin{array}{c}24.2 \\
(2.8)\end{array}$ \\
\hline Manganeso & $\begin{array}{c}597 \\
(122)\end{array}$ & $\begin{array}{c}477 \\
(135)\end{array}$ & $\begin{array}{l}481 \\
(88)\end{array}$ & $\begin{array}{c}804 \\
(234)\end{array}$ & $\begin{array}{l}597 \\
(89)\end{array}$ \\
\hline Hierro & $\begin{array}{l}152.0 \\
(22.4)\end{array}$ & $\begin{array}{c}94.3 \\
(12.8)\end{array}$ & $\begin{array}{l}176.0 \\
(38.9)\end{array}$ & $\begin{array}{c}298.1 \\
(26.0)\end{array}$ & $\begin{array}{l}130.9 \\
(21.6)\end{array}$ \\
\hline Aluminio & $\begin{array}{r}244.0 \\
(61)\end{array}$ & $\begin{array}{r}181.0 \\
(34)\end{array}$ & $\begin{array}{r}300.0 \\
\quad(86)\end{array}$ & $\begin{array}{l}205.0 \\
\quad(26)\end{array}$ & $\begin{array}{r}303.0 \\
(36)\end{array}$ \\
\hline
\end{tabular}


no son limitantes para el crecimiento de los árboleso Presentan bajas correlaciones con el crecimiento de los árboles y oscilan dentro de un intervalo de concentración adecuada para la especie. Respuestas a fertilización con estos nutrientes no son esperadas en las plantaciones de la región de estudio.

Para el N, se observa aumento en los contenidos del elemento con el aumento de la altura de los árboles. Sin embargo, los contenidos en los árboles de menor crecimiento se encuentran sobre el nivel de deficiencia definido por Kaul et ai. (1968). En los árboles de mayor altura, los contenidos están sobre el óptimo definido por Haag et ai. (1961). La correlación positiva entre el contenido de $\mathrm{N}$ y el crecimiento de los árboles indica una posible respuesta a aplicaciones de fertilizantes nitrogenados en plantaciones con los menores crecimientos.

La falta de correlación observada entre la cantidad de $\mathrm{N}$ aplicada como fertilizante mineral y la variación de los contenidos de $\mathrm{N}$ en las hojas en los diferentes sitios de plantación (cuadro 2) es debida, posiblemente, al tiempo transcurrido entre la aplicación deI fertilizante y la toma de muestras (a los 3 afios de edad). Durante este período, una parte del fertilizante puede haber sido absorbida, otra perdida por lixiviación y otra inmovilizada. Así, en ausencia del efecto de la fertilización, la única fuente de $\mathrm{N}$ en el suelo para los árboles es la. mineralización del N-orgánico por los microorganismos.

Los contenidos de $\mathrm{P}$ en las hojas de los árboles de todos los sitios estudiados se encuentran debajo del nivel óptimo citado en la literatura (Herbert y Schõnau, 1989). Para los árboles con alturas inferiores a $17 \mathrm{~m}$ se observan concentraciones inferiores a $1.0 \mathrm{mg} / \mathrm{g}$ M.S., mientras que en árboles con altura superior a $17 \mathrm{~m}$ las concentraciones se encuentran sobre este valor.

En relación a la fertilización, se puede observar el efecto positivo de la aplicación de P. Las correlaciones positivas observadas indican que el aumento en la oferta de este elemento en el suelo, a través de la fertilización en plantaciones con 3 aüos de edad, puede proporcionar una mayor absorción y, consecuentemente, un mayor crecimiento de los árboles, principalmente en los sitios donde .se observaron menores crecimientos.

Las concentraciones medias de $\mathrm{K}$ en las hojas, en todos los sitios, se encuentran debajo del valor óptimo citado por Herbert y Schõnau (1989) y Bellote (1990), o sea $7.5 \mathrm{mg} / \mathrm{g}$ M.S. De acuerdo con los datos de Kaul et ai. (1968), los árboles con altura bajo los $17 \mathrm{~m}$ se encuentran con deficiencia nutricional. Las correlaciones positivas y altamente significativas (cuadro 3) muestran que el $\mathrm{K}$ es el nutriente que más contribuye en explicar el crecimiento de los árboles.

\section{CUADRO 3}

Correlaciones (R) de los nutrientes en el suelo (nutr.lsuelo), en las hojas (nutr.lhojas) y altura de E. grandis eon 3 afios de edad $(\mathrm{n}=75)$.

Linearregressioncoefficientsmongnutrientconcentrations in soi!, in leaves, and for the averageheight of three-yearsold E. grandis.

$\begin{array}{lccc} & \text { Nutr.lhojas } & \text { Nutr.lsuelo } & \text { Nutr.lhojas } \\ \text { Nutriente } & \mathrm{x} & \mathrm{x} & \mathrm{x} \\ & \text { altura } & \text { altura } & \text { Nutr.lsuelo } \\ & & & \\ \text { Fósforo } & 0.478^{* * *} & 0.652^{* * *} & 0.446 * * * \\ \text { Potasio } & 0.860^{* * *} & 0.870^{* * *} & 0.785^{* * *} \\ \text { Magnesio } & 0.751 * * * & 0.761 * * * & 0.632 * * * \\ \text { Caleio } & -0.289^{*} & 0.635^{* * *} & 0.125 \\ \text { Aluminio } & -0.007 & 0.752^{* * *} & 0.341^{* *} \\ \text { Nitrógeno } & 0.555^{* * *} & - & - \\ \text { Azufre } & 0.407 * * * & - & - \\ \text { Boro } & -0.651^{* * *} & - & - \\ \text { Hierro } & -0.204 & - & - \\ \text { Manganeso } & -0.200 & - & - \\ \text { Zine } & 0.354 * * & - & - \\ & & & \\ * \text { (p }<0.05) & * *(\mathrm{p}<0.01) & * * *(\mathrm{p}<0.001) .\end{array}$

En relación a la fertilización, es posible observar cierta influencia de las cantidades de fertilizantes utilizadas sobre la altura de los árboles. Esta influencia no es mayor debido a la movilidad del $\mathrm{K}$ en el suelo $\mathrm{y}$, en el ejemplo del $\mathrm{N}$, al tiempo largo entre la época de fertilización y la toma de muestras. Los resultados sugieren aplicación de K en plantaciones con 3 afios de edad, especialmente en las áreas donde es menor la altura de los árboleso

El Mg es, después del $\mathrm{K}$, el elemento que más se relaciona con el crecimiento de los árboles. Contenidos mayores que $2.5 \mathrm{mg} / \mathrm{g}$ M.S. son observados solam ente en los árboles con mayor crecimiento. Los árboles en los sitios de menor crecimiento muestran deficiencia, de acuerdo con Kaul et ai. (1968). 
Las correlaciones positivas y altamente significativas encontradas para el $\mathrm{Mg}$, comprueban que este elemento es uno de los más importantes a ser considerado para aumentar el crecimiento de los árboles. Fertilizaciones complementarias con este elemento deben ser realizadas tanto en el momento de plantar como en plantaciones de 3 afios de edad, en todos los sitios considerados.

La concentración de AI en las hojas varía dentro de un intervalo bastante aceptable. Los árboles en todos los sitios estudiados no presentan problemas de toxicidad con este elemento, comprobando ser E. grandis una especie bastante tolerante a cantidades altas en el suelo.

La correlación significativa del contenido de AI en el suelo con el crecimiento de los árboles, como también como con los contenidos en las hojas, fue la esperada. Como muestra el cuadro 4, los sue] os estudiados son muy ricos en AI, elemento responsable por casi la totalidad de la saturación de bases en estos suelos. Como E. grandis es una especie tolerante a concentraciones altas de AI, se puede prever que cuanto mayor es el crecimiento de los árboles, mayor cantidad de AI es pasivamente absorbida. Resultados semejantes son descritos por Ferraz (1985) para sitios de Picea abies en la SeIva Negra, Alemania.

Los contenidos de B difieren mucho del encontrado en la literatura, tanto para concentraciones óptimas (32 mg/g, Herbert y Schõnau, 1989) como para concentraciones de deficiencia $(12 \mathrm{mg} / \mathrm{g}$, Rocha Filho et al., 1979). Las concentraciones observadas mantienen una correlación negativa con el crecimiento de los árboles, relación interpretada como casual y sin significado fisiológico. Los resultados demuestran un efecto de dilución de este elemento, causado por el crecimiento de los árboles.

Los contenidos de $\mathrm{Ca}$ en las hojas varían mucho, y demuestran una correlación floja y negativa con el crecimiento. La relación negativa como en el ejemplo del nutriente $B$, es también interpretada como un efecto de dilución, causado por el crecimiento de los árboles. Estos resultados demuestran, para las condiciones estudiadas, que la altura de los árboles no es dependi ente de los contenidos de $\mathrm{Ca}$ en las hajas. En caso que exista dependencia, posiblemente las hojas no sean el material vegetal más recomendado para ser utilizado en los análisis de diagnóstico.
CU ADRO 4

Contenidos de AI, capacidad de cambio de cationes (meq/g de sueio) y saturación de AI (\%).

Aluminium content, cation exchange capacity and aluminium saturation for the soils studied.

\begin{tabular}{lccrr} 
& \multicolumn{2}{c}{ Latosol } & Arenas & Cuarzosas \\
Contenidos & AI & CCC & AI & CCC \\
& & & & \\
Medio & 11.8 & 15.4 & 8.0 & 10.8 \\
Máximo & 13.2 & 19.7 & 9.8 &, 13.2 \\
Mínimo & 10.6 & 13.2 & 4.6 & 7.1 \\
& & & & \\
Saturación de AI & 90 & & & 92
\end{tabular}

CRECIMIENTO EN ALTURA DE LOS ARBOLES

a) Crecimiento en función de los nutrientes en las hajas. Las concentraciones de $\mathrm{K}$ y $\mathrm{Mg}$ en las hajas presentan correlación altamente significativa con la altura de los árboles. De esta forma se buscó una relación numérica entre estas variables a través de un modelo de regresión lineal múltiple, presentado en el cuadro 5.

Aunque los árboles dependan bastante deI suplemento de $\mathrm{K} \mathrm{y} \mathrm{Mg}$, el aumento en las concentraciones de $\mathrm{K}$ tiene un repercusión mayor en la altura de los árboles que el aumento de las concentraciones de Mg. Como muestra la ecuación del cuadro 5, para la menor concentración de $\mathrm{Mg}$ (1.1 $\mathrm{mg} / \mathrm{g}$ M.S.), una variación del $\mathrm{K}$ de 2.3 hasta 8.3 mg/g M.S. significa que los árboles pasan de $14 \mathrm{a}$ $21 \mathrm{~m}$ de altura media.

Los árboles con abastecimiento de $\mathrm{K}$ bajo el límite de deficiencia (Kaul et al., 1968) pueden legar a una altura máxima de $18 \mathrm{~m}$. Los árboles con abastecimiento óptimo, o sea $7.5 \mathrm{mg} / \mathrm{g} \mathrm{M.S.,}$ varían en altura desde $20 \mathrm{~m}$ (cuando la concentración de $\mathrm{Mg}$ es $1.1 \mathrm{mg} / \mathrm{g}$ M.S.) hasta $22.5 \mathrm{~m}$ (cuando la concentración de $\mathrm{Mg}$ es $3.2 \mathrm{mg} / \mathrm{g}$ MS).

Los resultados indicaron, para las condiciones de este trabajo, que la concentración adecuada de $\mathrm{Mg}$, definida en la literatura, no es suficiente para obtener incrementos máximos en crecimiento de los árboles. Esta solamente sería posible con contenidos de $\mathrm{Mg}$ en las hojas entre 2.6-3.2 mg/g M.S. Arboles con abastecimiento de $\mathrm{Mg}$ dentro de este intervalo y de $\mathrm{K}$ mayor o igual a $7.5 \mathrm{mg} / \mathrm{g}$ M.S. estarían óptimamente nutridos con estos elementos. 


\section{CUADRO 5}

Regresi6n múltiple entre la altura de los árboles (m) y los eontenidos de nutrientes minerales en hojas de E. grandis. Multiple regression between tree height and mineral nutrient content in leaves of E. grandis.

\section{Variables}

\section{Dependiente}

Altura de los árboles

Potasio
Magnesio
(Constante)

Independientes b

$\mathrm{R}^{2}$

0.740

0.762

1.199

0.686

0.229

207.491

9.839

$\mathrm{F}[72 ; 2 \mathrm{p}=0.011=4.91$

\section{CUADRO 6}

Regresi6n múltiple entre altura de los árboles (m) eon los eontenidos de $\mathrm{P}$ (mglg), $\mathrm{K}$ y AI (meqlg) dei suelo. Multiple regression between tree height and soi! nutrient content.

\begin{tabular}{llcccc}
\multicolumn{2}{c}{ Variables } & & & \\
Dependiente & Independientes & $\mathrm{b}$ & $\mathrm{R}^{2}$ & $\mathrm{~B}$ & $\mathrm{~F}$ \\
Altura de los & & & & & \\
árboles & Potasio & 8.813 & 0.756 & 0.335 & 226.646 \\
& Fósforo & 1.424 & 0.845 & 0.442 & 196.025 \\
& Aluminio & 0.432 & 0.886 & 0.407 & 184.754 \\
& (Constante) & 9.646 & & &
\end{tabular}

$\mathrm{F}[3 ; 71 \quad \mathrm{P}=0.01]=4.07$

b) Crecimiento en funciôn de los elementos mine rales en el suelo. La regresión múltiple entre las variables $\mathrm{P}, \mathrm{K}, \mathrm{Ca}, \mathrm{Mg}, \mathrm{AI}, \mathrm{H}$, materia orgánica, $\mathrm{pH}$, arena, arcilla dei suelo y la altura de los árboles demuestra que los contenidos de K, P Y AI en el suelo influencian significativamente el crecimiento (cuadro 6).

EI efecto positivo dei $\mathrm{K}$ y dei $\mathrm{P}$ es reforzado por una serie de trabajos de fertilización, los cuales demuestran mejores resultados para aplicaciones conjuntas de fertilizantes que contienen $\mathrm{K}$ y $\mathrm{P}$ en E. grandis (Malvos, 1983).

EI aumento en la oferta dei $\mathrm{K}$ del suelo resulta en mayor disponibilidad para las plantas y, consecuentemente, posibilidades mayores de incrementos en altura. Esto fue confirmado en los trabajos de fertilización con $\mathrm{K}$ realizados por Carvalho et ai. (1978) y Barros et ai. (1981).

Para el $\mathrm{P}$ en el suelo, ocurre una situación un poco diferente de aquella observada para el K. En este caso, el crecimiento de los árboles depende de los contenidos de $\mathrm{P}$ en el suelo, aunque sea baja la correlación entre $\mathrm{P}$ en las hojas y el crecimiento de los árboles, como también entre su oferta en el suelo y el contenido en las hojas (cuadro 3). Posiblemente, ésto se debe a las interacciones dei elemento con el sueio de los sitios estudiados. La suposición es que el aumento en la oferta de $\mathrm{P}$ para los árboles produzca un aumento en la absorción de $\mathrm{K}$ y $\mathrm{Mg}$, pues es conocido en la literatura pertinente el efecto dei $\mathrm{P}$ en la absorción de $\mathrm{Mg}$ por las plantas.

Aunque el AI sea una de las variables presentes en los análisis de regresión múltiple, con influencia significativa en el crecimiento de los árboles (cuadro 6), éste no tiene importancia fisiológica. Su relación con la altura de los árboles es debida, exclusivamente, a su altísima concentración en el 
suelo (cuadro 4), asociada con la alta tolerancia de E. grandis con este elemento.

Los resultados obtenidos indican que el crecimiento de los árboles en sitios de baja productividad, en los sitios de estudio, puede ser mejorado tanto con el aumento de los contenidos de $\mathrm{K}$ y $\mathrm{Mg}$ en los árboles (cuadro 5), como con el aumento en la oferta de $\mathrm{P}$ y $\mathrm{K}$ en el suelo (cuadro 6).

\section{CONCLUSIONES}

Las concentraciones de $\mathrm{S}, \mathrm{Fe}, \mathrm{Mn}$ y $\mathrm{Zn}$ en las hojas de los árboles no son limitantes para el crecimiento deI E. grandis. Las concentraciones de $\mathrm{P}$ en las hojas, sin embargo, se encuentran bajo el valor óptimo establecido en la literatura.

EI K Y el Mg son los nutrientes más limitantes deI crecimiento. Arboles con contenidos en las hojas entre 2.6-3.2 mg/g de M.S., de $\mathrm{Mg}$ y de $\mathrm{K}$ mayor o igual a $7.5 \mathrm{mg} / \mathrm{g}$ de M.S. se encuentran óptimamente nutridos con estos elementos.

Tanto para el Ca, como para el B de las hojas, fueron observados efectos de dilución causados por un crecimiento mayor de los árboles.

La oferta de $\mathrm{K}$ en el suelo es el factor más limitante tanto para el crecimiento de los árboles como para su nutrición adecuada.

Los resultados indican posibilidades de incrementos en productividad de madera con el aumento en la aplicación de fertilizantes con $\mathrm{N}, \mathrm{P}, \mathrm{K} \mathrm{YMg}$.

\section{BIBLIOGRAFIA}

BARROS, N.F., 1.M. BRAGA, R.M. BRANDI, B.V. DEFELIPO. 1981. "Produção de eucalipto em solo de Cerrados en resposta a aplicação de NPK, B e Zn", Revista Arvore, Viçosa 5 (I): 90-103.

BELLOTE. A.F.J. 1990. "Naehrelementversorgung und Wuchsleistung von geduengten Eucaiyptus grandis Plantagen im Cerrado von São Paulo (Brasil ien)",
Freiburger Bodenkundliche Abhandlungen. Freiburg, 26 $1-159$.

CARVALHO, C.M., E.S. BAENA, C.J. COUTINHO, M. FREITAS, C.A. FERREIRA. 1978. "Estudos das relações $B / K$ e $B I C a$ na cultura de E. saligna Smith. (Resultados preliminares)". Silvicultura, São Paulo, 13: 264-266.

DEFELIPO, B.V., V.H. ALVAREZ, L. COUTO, 1.C. FERNANDES. 1979. "Estudo de micronutrientes em solos de Cerrado de Minas Gerais", Boi. Técnico SIF, Viçosa, 2: 15-26.

FERRAZ, 1.B.S. 1985. Standortsbedingungen, Bioelementversorgung und Wuchsleistung von Fichtenbestãnden (Picea abies Karst) des Sudschwarzaldes. Forstwissenschaftlichen Fakultat Universitãt Freiburg.

GARLIPP, R.C.D., EA BALLONI. 1980a. "Estudo sobre o efeito da omissão de nutrientes em plantios de E. grandis", Boletim Informativo IPEF, Piracicaba, 8 (26): 21-22.

GARLIPP, R.C.D., EA BALLONI. 1980b. "Estudo sobre a influencia da fertilização fosfatada no desenvolvimento de E. grandis", Boletim Informativo IPEF, Piracicaba, 8 (26): 25-29.

GONÇALVES, 1.C., A.S. DINIZ. 1981. "Efeito da época e forma de aplicação de fertilizantes no plantio de E. saligna", Boletim Informativo IPEF, Piracicaba 9 (28): 21-23.

HAAG, H.P., F.A.F. MELLO, M.O.C. BRASIL SOBRINHO, W.R. ACORSI, E. MALA VOLTA, S. ARZOLLA. 1961. "Estudo sobre a alimentação mineral do Eucalyptus", En: Conferência Mundial do Eucalipto. São Paulo. Rei. e documentos. São Paulo, FAO, pp. 926-932.

HERBERT, MA, A.P.G. SCHONAU. 1989. Fertilizing commercial forest species in southern Africa. Research progress and problems. En: Symposium: "Mineralversorgung tropischer Waldbãume". Bayreuth.

KAUL, O.N., P.B.L. SRIVASTAVA, V.N. TANDON. 1968 "Nutrition studies on Eucalvptus. Ill. Diagnosis of mineral deficiencies in Eucalyptus grandis seedlings, Indian Forester 94 (11): 83 I-834.

MAL VOS, C. 1983. "First results on fertilization trials given to Eucalyptus plantations in Madagascar", Silvicultura 32 625-626.

MOLL, W. 1964. "Ntihrstoffversorgung von Fichtenbestãnden im Nordschwarzwald", Schriftreihe d. Forstl. Abt. d. Univ. Freiburg. BL V München 4: 252-265.

NOVAIS, R.F., 1.M. GOMES, E.E.L. BORGES, D. ROCHA. 1980. "Cal agem e adubação na produção de mudas de eucalipto. (E. grandis). II-efeitos da cal agem, do $\mathrm{N}$ e do superfosfato simples", Revista Arvore. Viçosa 4 (I): 1-13.

ROCHA FILHO, 1.V.C., H.P. HAAG, G.D. OLIVEIRA. 1979. "Influência do Boro no crescimento e na composição química de E. grandis", Anais da ESALQ, Piracicaba, 36 (I): 139-151.

TRÜBY, P., E. ALDINGER. 1989. "Eine Methode zur Bestimmung der austauschbaren Kationen in Waldboden", Z. P.flanzenerniihr. Bodenk. 152 (I): 301-306. 